\title{
High-Resolution Photography of Snowflakes with a Field Microscope
}

\author{
3ric Johanson, ${ }^{1 *}$ Charles Krebs, ${ }^{1}$ and Nathan P. Myhrvold ${ }^{1,2}$
}

${ }^{1}$ Intellectual Ventures, $3150139^{\text {th }}$ Ave SE, Bellevue, WA 98005

${ }^{2}$ Modernist Cuisine Gallery, $14031^{\text {st }}$ Ave, Seattle, WA 98101

*3ricj@intven.com

\begin{abstract}
Snowflakes are a natural subject for microscopy, but the conditions under which they form, and the minutes-long working time imposed by sublimation, are substantial technical barriers to field microscopy of snow crystals. Building on the work of earlier pioneers, we developed a portable, actively cooled, semi-automated microscope system capable of making z-stacked images of individual snowflakes and small groups of crystal specimens at ultrahigh resolution. Field trials of the system in Alaska and Canada produced encouraging results and also suggested directions for further improvement.
\end{abstract}

Keywords: snowflake, snow crystal, field microscopy, thermal stabilization, ultrahigh resolution

\section{Introduction}

At some point in life, most people who experience winter develop a fascination with snow and admire the intricate, seemingly innumerable patterns of snowflakes. These iconic natural crystals form by direct deposition of water vapor onto nucleation seeds and, under exceptional conditions, can grow to as much as a centimeter in diameter. Though the crystals frequently aggregate to form composite flakes, individual snow crystals are typically $1-5 \mathrm{~mm}$ in diameter and at least $10 \mu \mathrm{m}$ thick. Fragile, transparent, and short-lived in their nascent state, snowflakes are attractive but highly challenging photographic subjects that span the overlapping domains of macrophotography and microscopy.

While much progress has been made in using molecular dynamics simulations and other computational techniques to model how snowflakes develop their characteristic faceted, branching crystal structures [1], imaging the submicron-size features that are so distinctive in natural snowflakes presents sufficient technical obstacles that only a few pioneering microscopists have published extensive collections of snowflake photomicrographs.

Wilson Bentley, a meteorologist and early innovator in photomicrography, is believed to have made the first photomicrographs of individual snow crystals in 1885. Using a bellows camera attached to a microscope objective and illuminated by daylight, Bentley persisted through multiple failures until he devised a dry-plate photographic system (later converted to use film) for imaging snowflakes [2]. Working in an unheated room in his farmhouse in Jericho, Vermont, Bentley produced more than 400 photomicrographs of snowflakes over 13 years before publishing his first article on the work [3]. To achieve a darkfield effect, Bentley painstakingly scraped away the emulsion from around each snowflake image in his negatives [4]. In a book first published just a month before his death from pneumonia in 1931 [5], Bentley showed the world just how dynamic these crystals are.

More recently, physicist Kenneth Libbrecht at Caltech has developed macrophotography systems and techniques to make full-color, high-resolution snowflake images, which he has published in a series of books [6-10]. Libbrecht has demonstrated methods for growing snowflakes in the laboratory and has used these artificial crystals to study the dynamics of snow crystal formation and the aerodynamics of falling snow. These results, along with numerous useful methods for snowflake photography, are described in a 2019 monograph [11].

Many other workers have produced snowflake photographs, but Bentley and Libbrecht stand out for the volume and high quality of their work. Both refined techniques of transmitted light microscopy to record snowflakes-the same approach taken here. Another group of photographers, working in the tradition of macrophotography, have used reflected light to photograph snowflakes or snowflake casts $[12,13]$. The equipment described here can be modified to use reflected light, and we hope to do that at some point in the future.

We developed equipment, software, and techniques applicable to snowflake microscopy through previous work at our laboratory, creating z-stacked photomicrographs of foods and vitamin crystals at sufficiently high resolution to permit printing at sizes up to $2 \mathrm{~m}$ wide. In 2019, we constructed a portable microscopy system designed specifically for field imaging of snowflakes at ultrahigh resolutions made possible by new medium-format, high-pixel-count digital sensors (Figures 1 and 2).

Although a sophisticated system like the one we constructed is not necessary to make high-quality snowflake photos at standard resolutions, our aim was to create a semiautomated field microscope that reliably images snow crystals at ultrahigh resolution and in enough focal planes to perform Z-stacking, without relying on luck and patience.

Our field trials of the system in 2020 produced the highestresolution photographs of snowflakes published to date. Some of these images received widespread media attention [14-16]. We describe here how our system addresses many of the technical challenges particular to snowflake field microscopy. We also comment on directions for future improvement in snow-crystal imaging.

\section{Challenges of Snowflake Microscopy in the Field}

Writing in 1922, Bentley noted the central obstacle to imaging individual snow crystals: the very short working time before the crystal degrades from sublimation.

The utmost haste must be used, for a snow crystal is often exceedingly tiny, and frequently not thicker than heavy paper. Furthermore, once these bits of pure beauty are isolated, evaporation (not melting) soon wears them away, so that, even in zero weather, they last but a few minutes [4]. 
Radiation hard

\section{unsaturated \\ Zero Loss Peak}

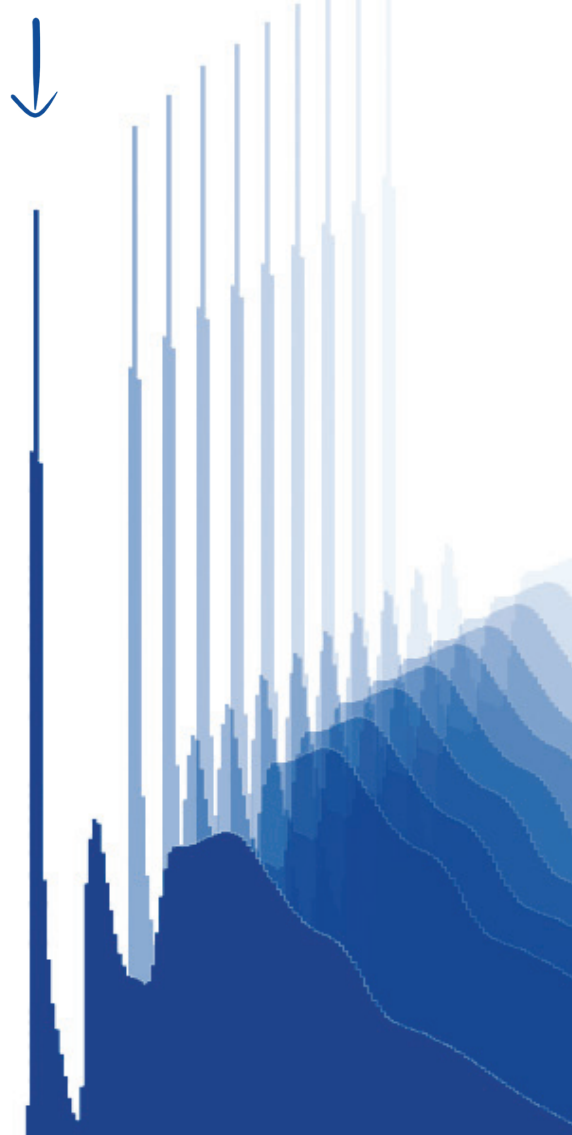

High dynamic range

over seven orders of magnitude

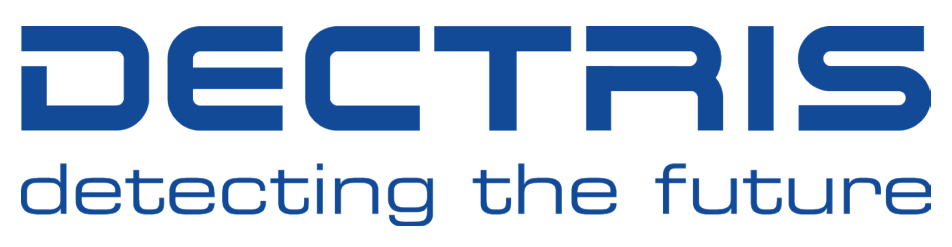

The highest frame rate

up to 18.000 frames

per second

Noise-free detection

single-electron counting across the whole spectrum

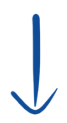

Make the most of electron microscopy with hybrid-pixel detectors

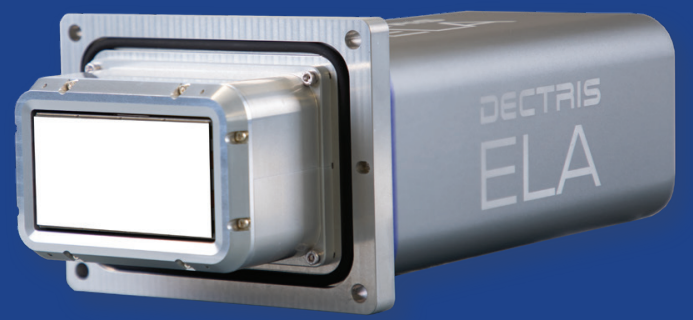




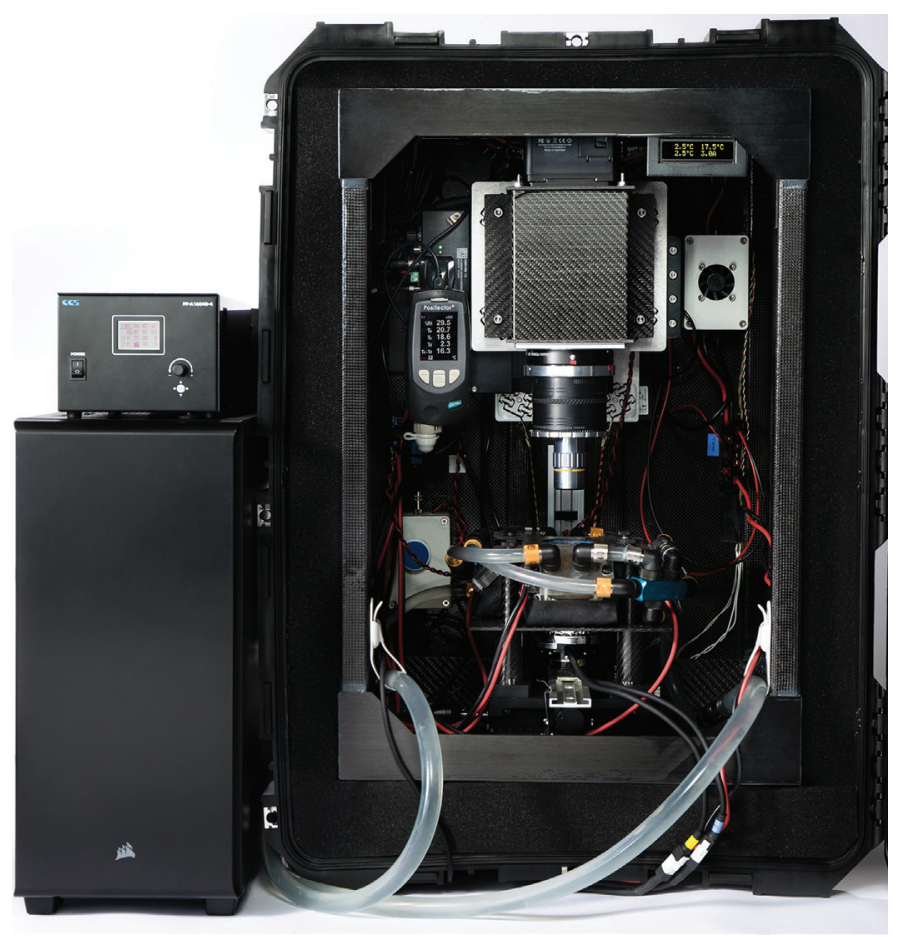

Figure 1: Snowflake field microscopy system. The optical train, stage, sensors, and most control electronics are fixed to a carbon-fiber frame $44 \mathrm{~cm}$ by $65 \mathrm{~cm}$ ), which fits within a foam-lined, ruggedized case for transport and field deployment. Connections to the external heat exchanger, LED strobe control, and a laptop can be made without removing the microscope from the case.

Bentley made an important point. One might easily expect that melting would be the primary concern, but melting is easy to avoid by chilling the equipment to below $0^{\circ} \mathrm{C}$. Sublimation, on the other hand, is a less predictable process that depends on both temperature and the relative humidity in the immediate vicinity of the snowflake. Sublimation causes the loss of fine details, the rounding of sharp edges, and general degradation of the snowflake [17].

Bentley made just one image per snowflake, whereas our aim is to capture, in focus, every part of each snowflake in a $5 \mathrm{~mm}$ field-of-view at a magnification up to $24 \times$. In images that include multiple crystals, the snowflakes may not be exactly coplanar, and z-stacking of the scene may thus require up to 100 digital images taken in focal planes separated by less than $1 \mu \mathrm{m}$. This requirement translates to a need for very rapid, precise, and fully automated focal and shutter control.

In order to maximize the working time, every piece of equipment that touches the snowflake must be at or below ambient temperature-and that air temperature should be far below $0^{\circ} \mathrm{C}$ but well above the local dew point at which frost forms on surfaces. Winter temperatures at some of the best locations for field microscopy of snowflakes-such as Fairbanks, Alaska; Timmins, Ontario; and Yellowknife, Northwest Territories-range from $-20^{\circ} \mathrm{C}$ to $-35^{\circ} \mathrm{C}$. Such low temperatures pose obvious challenges for human operators and electronics, but perhaps the more significant impact is the very long time that passive equipment requires to reach thermal equilibrium after it is brought outdoors. Metals typically used in microscope frames have high coefficients of thermal expansion
(CTE) $\left(23.6 \mu \mathrm{m} / \mathrm{m}^{\circ}{ }^{\circ} \mathrm{C}\right.$ for aluminum $)$ that result in size changes comparable to the thickness of a snowflake at the $\sim 1 \mathrm{~m}$ height of our microscope and the $\sim 40^{\circ} \mathrm{C}$ difference between indoor and outdoor temperatures.

Ice crystals are transparent and thus require specialized lighting techniques to highlight in color against dark fields, light fields, or colored backgrounds. Conventional microscope condensers typically lack the field-of-view necessary to capture full snowflakes, and their light sources generate significant heat, reducing the working time.

An additional challenge arises from our goal to maximize the meaningful resolution of the images, which is related to, but distinct from, the pixel size of the images [18], while simultaneously imaging a field-of-view large enough to contain several snowflakes. High numerical aperture (NA) objectives yield high resolution at the sensor plane, but they constrain the field-of-view and produce image circles too small to fill the large digital sensors now available. An optimized system should balance the NA of the objective with the size of the sensor, such that the maximum number of sensor pixels are used and are sufficient to cover the diffraction-limited Airy discs produced by the optics [18].

Finally, snow is a product of weather, and the weather conditions conducive to high-quality snowflake microscopy are fleeting and difficult to predict. Portability of the system and speed of setup and calibration are thus crucial features in a field microscope for this application. It took Wilson Bentley 13 years of steady work to acquire 400 snowflake images; our aim is to improve on that pace by two orders of magnitude.

\section{Stability and High Resolution at Thirty Below}

Each part of our snowflake microscopy system was customized, often in several ways, to meet the challenges described above (Figures 1 and 2). The frame for the microscope was fabricated from carbon-fiber tubing, which offers high stiffness and strength at low weight and a low CTE of $0.8 \mu \mathrm{m} / \mathrm{m}-{ }^{\circ} \mathrm{C}$, about 30 times less than that of aluminum. The carbon-fiber frame improves portability and requires no prechilling, as it can equilibrate from room temperature to $-30^{\circ} \mathrm{C}$ in about 30 minutes.

The frame supports the optical train through a directdrive linear stage (Zaber X-LDM-060C). To maintain compliance and reduce stresses induced by the large difference in CTE between the aluminum stage and the carbon-composite frame and optical tube, we fabricated flexures from an aluminum plate.

A medium-format camera back (PhaseOne IQ3) tops the optical train. With a 100-megapixel sensor measuring $53.7 \mathrm{~mm}$ by $40.4 \mathrm{~mm}$, this camera can capture ultrahigh-resolution images by virtue of the sensor size relative to the size of the Airy disc produced by diffraction from the objectives (Mitutoyo Series Plan APO). A camera with a smaller sensor-even a fullframe $35 \mathrm{~mm}(24 \times 36 \mathrm{~mm})$ sensor-cannot compete, regardless of its native resolution or the use of resolution-enhancing techniques such as pixel shifting. The details recorded by the sensor are limited by the fixed size of the Airy disk, which allows fewer details from a smaller sensor [18].

Tests for chromatic aberration, center sharpness, edge sharpness, and vignetting on more than 30 configurations 


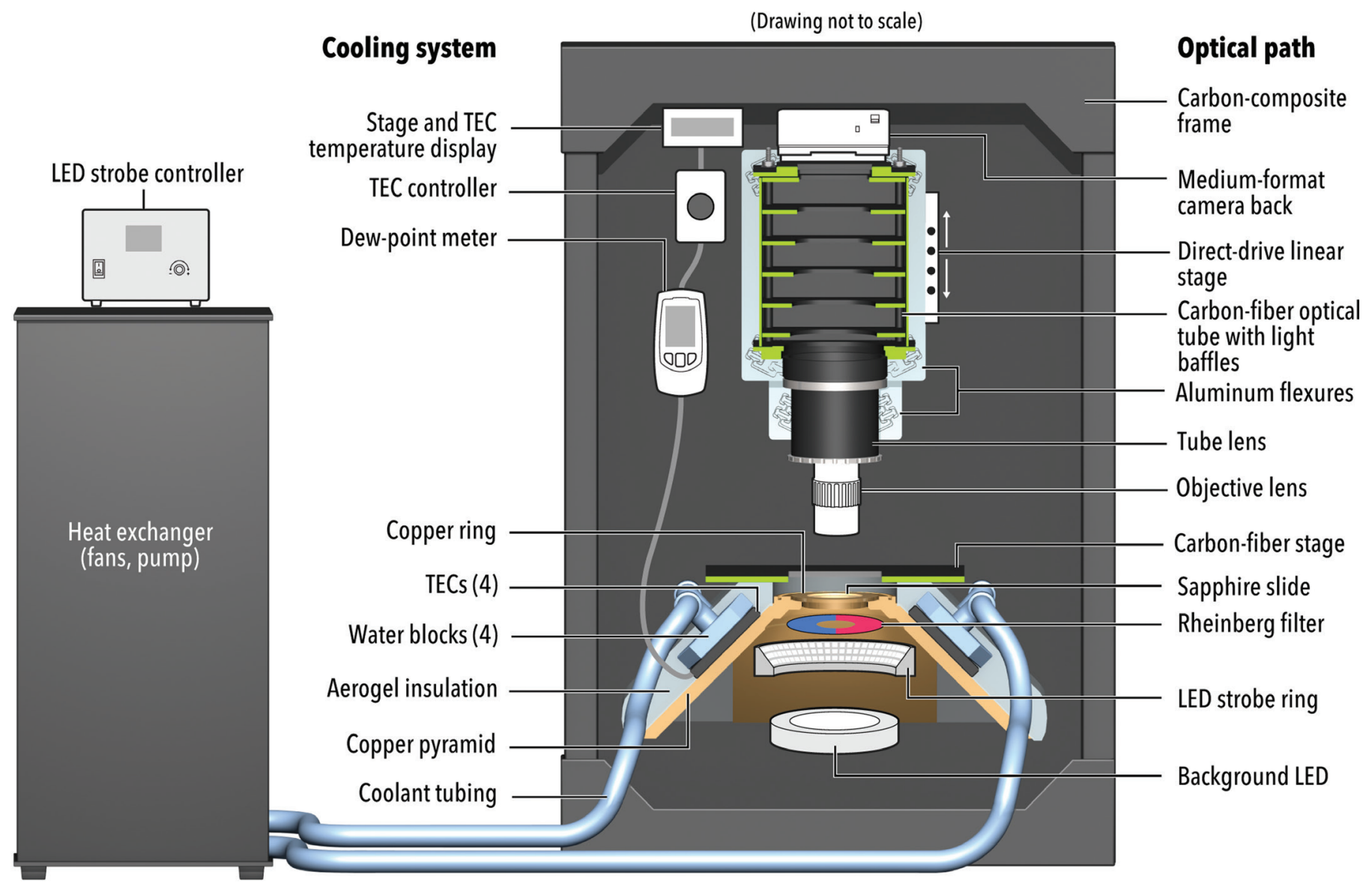

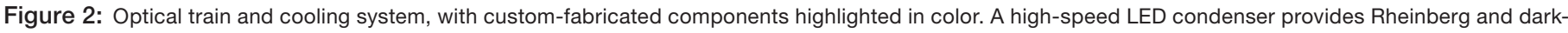

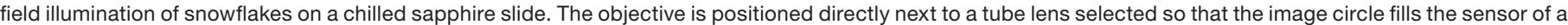

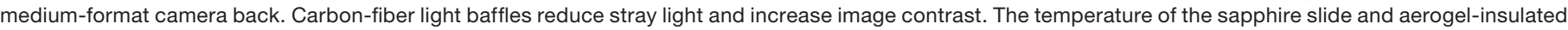

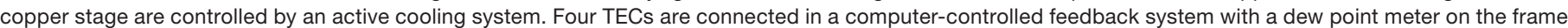

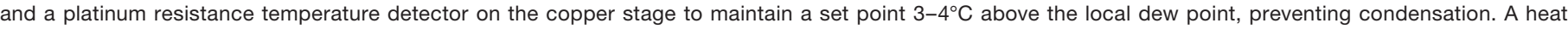
exchanger circulates potassium-formate-based fluid around the TECs to remove excess heat from the system.

led us to select a macro lens (Raynox DCR-5320PRO) to use as a tube lens. Although the specifications for the Mitutoyo objectives guarantee only a small $(\sim 30 \mathrm{~mm})$ image circle, we found that with this tube lens the image circle easily covers the $\sim 67 \mathrm{~mm}$ diameter required to illuminate all pixels of the PhaseOne sensor. We fabricated five carbon-fiber baffles, designed so that stray light must bounce at least three times in order to hit the sensor, increasing system contrast. With $5 \times$ to $10 \times$ objectives, the optical train produces Airy discs at the sensor that are $\sim 14 \mu \mathrm{m}$ in diameter, which are well covered by the sensor's $4.6 \mu \mathrm{m}$ pixels.

To avoid a lamp-house and condensers that would heat the snowflakes and reduce working time, we illuminate samples using high-intensity LEDs capable of pulses as brief as $1 \mu \mathrm{s}$, shorter than that of conventional flash units. The use of compact pulsed LEDs also shortens the optical path and eliminates motion blur. Rheinberg filters positioned over the LEDs allow us to highlight the detailed structure of the snow crystals in contrasting colors against brightfield and darkfield backgrounds of various colors (Figures 3-8). The software-controlled lighting system allows for rapid changes in brightness and pulse duration.

\section{Catching Snowflakes and Keeping Them Cool}

Much as Bentley did a century ago, we capture snowflakes on a large black surface (foam core) at ambient temperature and then transfer selected crystals to the microscope slide using a small sable brush, whose bristles pick up an electrostatic charge from the air that attracts snow crystals.

By the time we select a snowflake for imaging, its countdown to sublimation has already begun. Any object that delivers heat to the crystal accelerates the countdown, so our system is designed to chill the slide to below ambient temperature and hold it there while the photography proceeds.

Artificial sapphire slides hold the specimens. This material has seven times the thermal conductivity of glass and much greater hardness, so the slides do not scratch when brushed clean by a glove, and they cool quickly when placed on the cold stage. The cold stage is a truncated tetrahedral pyramid made from $1 \mathrm{~kg}$ of copper plate, which is insulated with aerogel and covered with an outer layer of carbon composite. A ring of copper holds the slide.

The stage temperature is controlled by a closed-loop feedback system that incorporates readings of the local dew point from a commercial sensor (DeFelsko PosiTector DPM). 


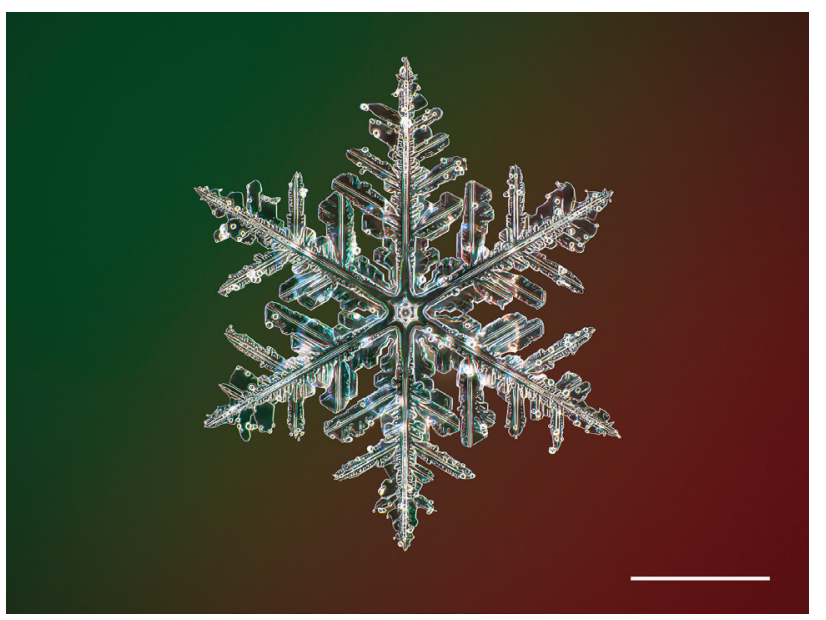

Figure 3: Stellar dendrite snowflake collected in Yellowknife, Northwest Territories, in March 2020. This 11,224 by 8,420 pixel, z-stacked photomicrograph (effective magnification $9 \times$ ) was created from 90 images captured at regularly spaced focal planes. Scale bar, $1 \mathrm{~mm}$. Photos OModernist Cuisine Gallery, LLC.

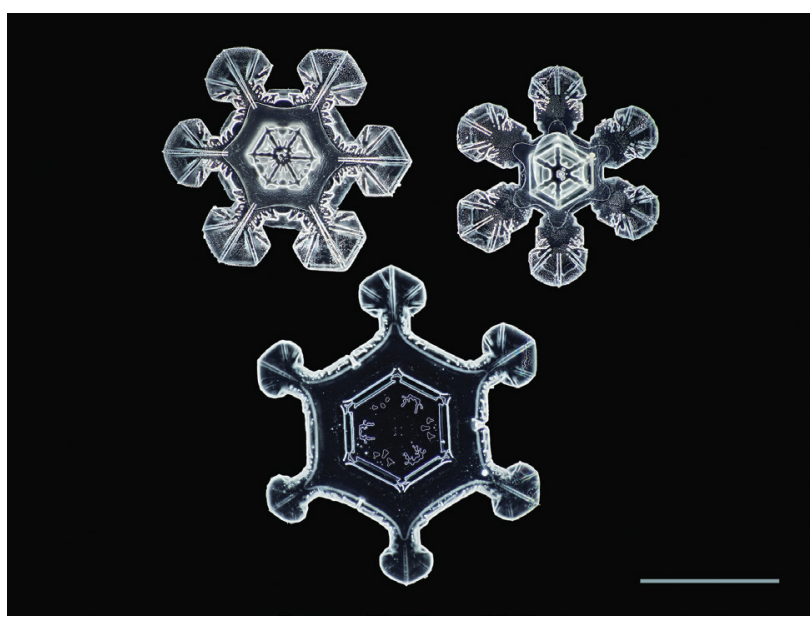

Figure 4: Three plate-like snowflakes collected in Fairbanks, Alaska in March 2020. $12 \times$ magnification, 11,247 by 8,437 pixels, 88 focal planes. Scale bar, $1 \mathrm{~mm}$.

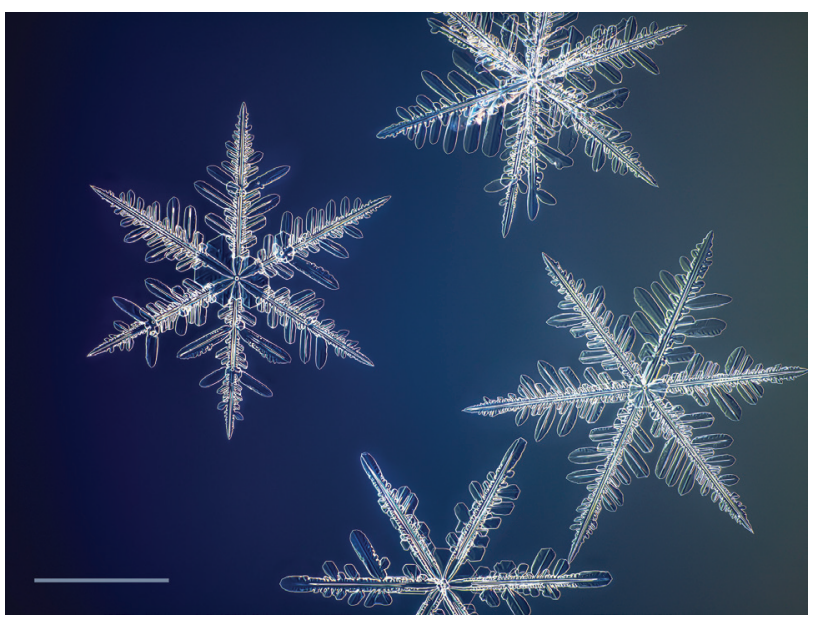

Figure 5: Two complete and two partial stellar dendrite snowflakes collected in Yellowknife in March 2020. 9x magnification, 11,608 by 8,708 pixels, 120 focal planes. Scale bar, $1 \mathrm{~mm}$.
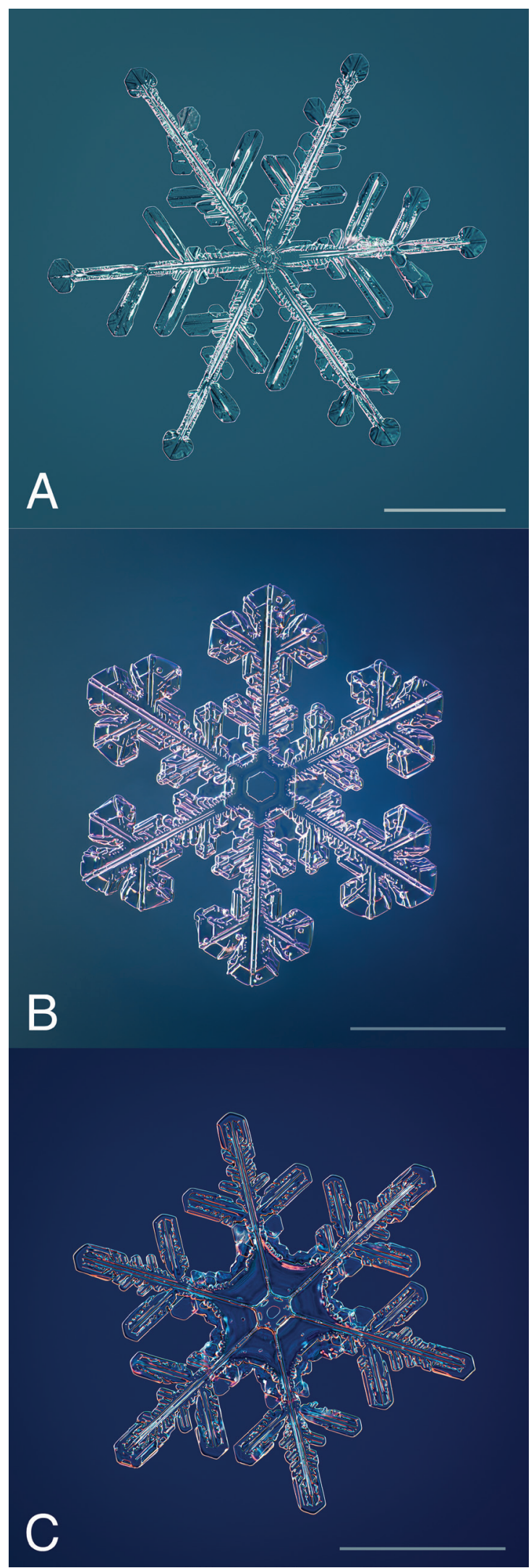

Figure 6: A trio of snowflakes collected in Yellowknife, Fairbanks, and Timmins, Ontario in February and March 2020. A. 8,336 by 8,336 pixels as cropped, 30 images at $9 \times$ magnification. B. 8,708 by 8,708 pixels, 77 images at $12 \times$ magnification. C. 8,145 by 8,145 pixels, 77 images at $12 \times$ magnification. Scale bars, $1 \mathrm{~mm}$. 


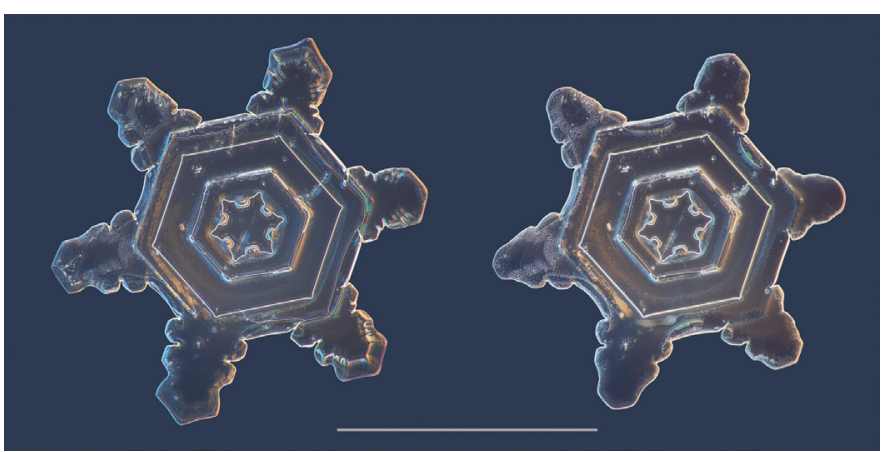

Figure 7: Two images of the same snowflake made 11.7 minutes apart. Even on a stage chilled to well below the freezing point, sublimation quickly rounds off sharp features from the outside inward, essentially recapitulating the growth of the crystal in reverse. $24 \times$ magnification, 77 focal planes. Scale bar, $1 \mathrm{~mm}$. Photos @Modernist Cuisine Gallery, LLC.

To avoid condensation or frost forming on the slide, the system selects a target stage temperature that is $3-4^{\circ} \mathrm{C}$ above the dew point-but still well below $0^{\circ} \mathrm{C}$. Thermal-control software, running on a USB-connected laptop, activates Peltier thermoelectric coolers (TECs) mounted on the four sides of the copper pyramid as needed to maintain the target temperature.

Heat is removed from the hot side of the TECs by water blocks through which coolant circulates. We found that marinegrade antifreeze, despite ratings down to $-70^{\circ} \mathrm{C}$, becomes slushy at the subzero temperatures common at our field locations. Instead, we use a potassium-formate coolant (Dynalene), which retains low viscosity in our operating regime. The coolant pump is housed externally in a heat-exchanger system, adapted from parts designed for water-cooled computers, that also includes fans to discharge excess heat from the system. Aerogel and foam insulation around the copper stage further enhance the system's thermal stability.

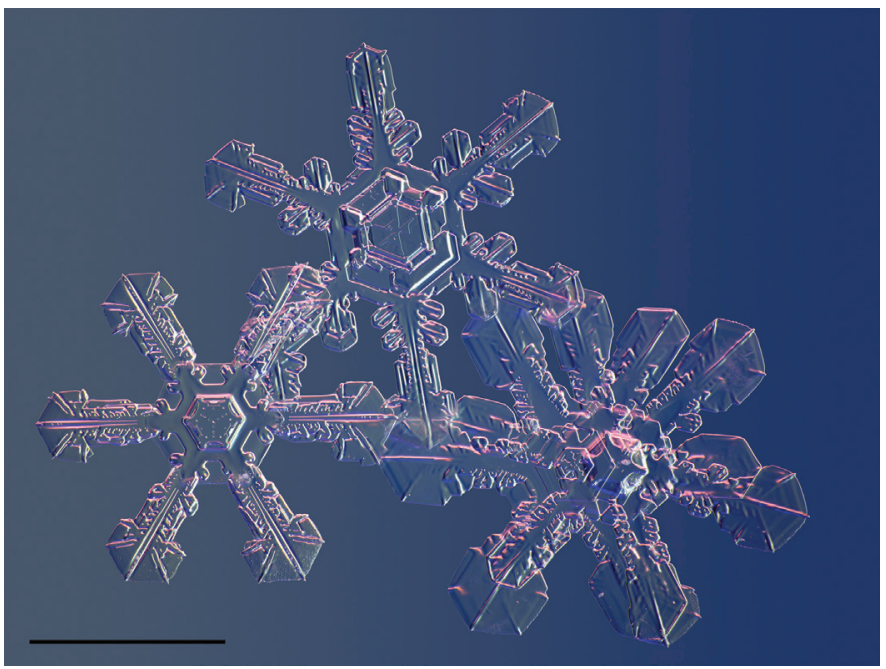

Figure 8: Snowflakes colliding. Snow crystals tend to tangle with one another as they fall. Arranging several in a frame can also be complicated by the repulsive electrostatic charge imparted by the brush used to transfer them to the slide. Some crystals-like the one seen here, end-on, at bottom right-form in 3-D structures shaped like two wheels connected by an axle. Scale bar, $1 \mathrm{~mm}$.

\section{Precision Focus Stacking at High Speed}

Resolution in microscopy comes at the cost of a shallow depth-of-field. The objectives we selected offer excellent resolution, but some have less than $3.5 \mu \mathrm{m}$ of usable depth-of-field. Because snowflakes can exceed $10 \mu \mathrm{m}$ in thickness and rarely lie flat, we typically take dozens of images at regularly spaced focal planes and then use z-stacking software to assemble a final image in which every pixel is in focus.

At the highest magnification we use, the $\mathrm{z}$ separation required is $0.7 \mu \mathrm{m}$. That is a challenge to achieve with a system such as this, in which the entire optical column moves while being exposed to temperatures far below zero. We are able to reliably achieve vertical stepping with such precision by using a linear stage driven by voice coils. Under the control of custom software, the drive system can rapidly shift the focal plane to any specified vertical position within a $60 \mathrm{~mm}$ range of travel with full-travel accuracy of $1 \mu \mathrm{m}$ and repeatability better than $0.08 \mu \mathrm{m}$.

In practice, many of our photographs required capture of over 100 focal planes because they included multiple snowflakes at various angles of tilt. The high speed of the automated focus-adjustment and shutter-control system allows us to complete such series within the few minutes available before the snowflakes degrade by sublimation (Figure 7). Commercial z-stacking software packages (Helicon Focus, Zerene Stacker) are used to process image series and produce a final photography that has effectively infinite depth-of-field.

\section{Results and Future Directions}

Initial trials of our snowflake microscope were held during the winter of 2019-2020 at field sites in Fairbanks, Yellowknife, and Timmons. During the trials, we encountered difficulties of several kinds with the system that spurred us to make refinements, particularly to the temperature control of the slide and the cold stage on which it rests. Adding ambient dew point sensing to the closed-loop temperature control and tuning the system to maintain temperatures $3-4^{\circ} \mathrm{C}$ above the local dew point greatly reduced issues with sublimation and condensation, maximizing the working time with our snowflake specimens. We were able, in the later trials, to routinely make multifocal image sets with up to 100 focal planes for dozens of snowflakes in the course of a day. Such high throughput is helpful because snowflakes are finicky subjects, difficult to arrange well on the slide (Figure 8).

We believe there remains much room for further improvement in field-capable snowflake microscopes, however. Additional capabilities to control humidity, increase resolution, and broaden the range of illumination modes would open new avenues for capturing these awe-inspiring creations of nature in all of their wondrous detail.

\section{Acknowledgments}

Wayt Gibbs provided editorial assistance for this article. George Retseck, Ross Ellingson, and Nate Paradiso assisted with diagrams and photography. Scott Heimendinger assisted with design of system components, and custom fabrication work was performed by the Intellectual Ventures machine shop. Toni Dysert assisted with field logistics. Joel Schweitzer assisted with software and field operations. 


\section{References}

[1] KG Libbrecht, Ann Rev Mater Res 47 (2017) https://doi. org/10.1146/annurev-matsci-070616-124135.

[2] DC Blanchard, Weatherwise 23 (1970) doi.org/10.1080/00 431672.1970 .9932907$.

[3] WA Bentley and GH Perkins, Pop Sci Mon 53 (May 1898) 15.

[4] WA Bentley, Pop Mech 37 (1922) 309-12.

[5] WA Bentley and WJ Humphreys, Snow Crystals, Dover Publications, New York, 1962.

[6] KG Libbrecht, Ken Libbrecht's Field Guide to Snowflakes, MBI Publishing Co., St Paul, MN, 2006.

[7] KG Libbrecht, Snowflakes: Featuring the Amazing MicroPhotography of Kenneth Libbrecht, Voyageur Press, Minneapolis, MN, 2008.

[8] KG Libbrecht, The Secret Life of a Snowflake: An Up-Close Look at the Art \& Science of Snowflakes, Voyageur Press, Minneapolis, MN, 2009.

[9] KG Libbrecht, The Art of the Snowflake: A Photographic Album, Voyageur Press, Minneapolis, MN, 2014.

[10] KG Libbrecht and R Wing, The Snowflake: Winter's Frozen Artistry, Voyageur Press, Minneapolis, MN, 2015.

[11] KG Libbrecht, Snow Crystals, 2019, arXiv.org/abs/ 1910.06389.

[12] Alexey Kljatov photography, "Snowflake Macro Photography." https://alexey-kljatov.pixels.com/blogs/2-snowflakemacro-photography.html (accessed May 12, 2021).
[13] D Komarechka, Sky Crystals: Unraveling the Mysteries of Snowflakes, Don Komarechka Photography, Barrie, Ontario, Canada, 2013.

[14] K Chang, "Snowflakes as You've Never Seen Them Before," New York Times, Mar. 3, 2021. https://www.nytimes. com/2021/03/10/science/snowflakes-photos-nathanmyhrvold.html.

[15] J Nalewicki, "These Are the Highest Resolution Photos Ever Taken of Snowflakes," Smithsonian Magazine, Jan. 27, 2021. https://www.smithsonianmag.com/innovation/ these-are-highest-resolution-photos-ever-taken-snowflakes-180976710.

[16] J Koetsier, "This is the Highest Resolution Snowflake Photo Ever," Forbes Magazine, Feb. 12, 2020. https://www. forbes.com/sites/johnkoetsier/2020/12/02/this-is-thehighest-resolution-snowflake-photo-ever.

[17] E Jambon-Puillet et al., Nat Commun 9 (2018) doi. org/10.1038/s41467-018-06689-x.

[18] NP Myhrvold, "More Pixels Do Not Always Yield Better Resolution-Especially When Photographing Small Things, Like Snowflakes," Medium, Feb. 27, 2021. https:// nathanpmyhrvold.medium.com/more-pixels-do-notalways-yield-better-resolution-especially-when-photographing-small-things-1fd3f07aa595.

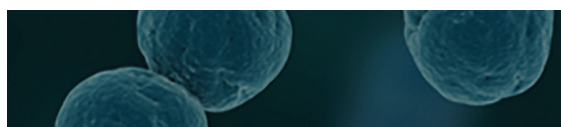

Do you have more jobs than capacity at your high-performance microscopes? Adding a ReManufactured Dual Beam, FIB, SEM or TEM may be the solution that helps you better manage the usage of your premium instruments.

\section{Free Your Premium Microscopes for Your Most Demanding Research}

TSS has you covered with service, spare parts and accessories for legacy instruments, even if you didn't purchase from us. And we now offer an Analytical Service Lab, too.

Browse our currently available inventory: www.tssmicroscopy.com/instruments

Or contact us to request a particular instrument and we will see if we can get it for you.

sales • service • analytical lab • parts • accessories electron microscopes re-visioned

USA Office: + 1866 TSS 2003 www.tssmicroscopy.com
Taiwan Office: +886 [0]916 942800 www.tssmicroscopy.com.tw 


\section{Transform Your Electron Microscope}

Into a Powerful, Nanoscale Mechanical Testing Chamber
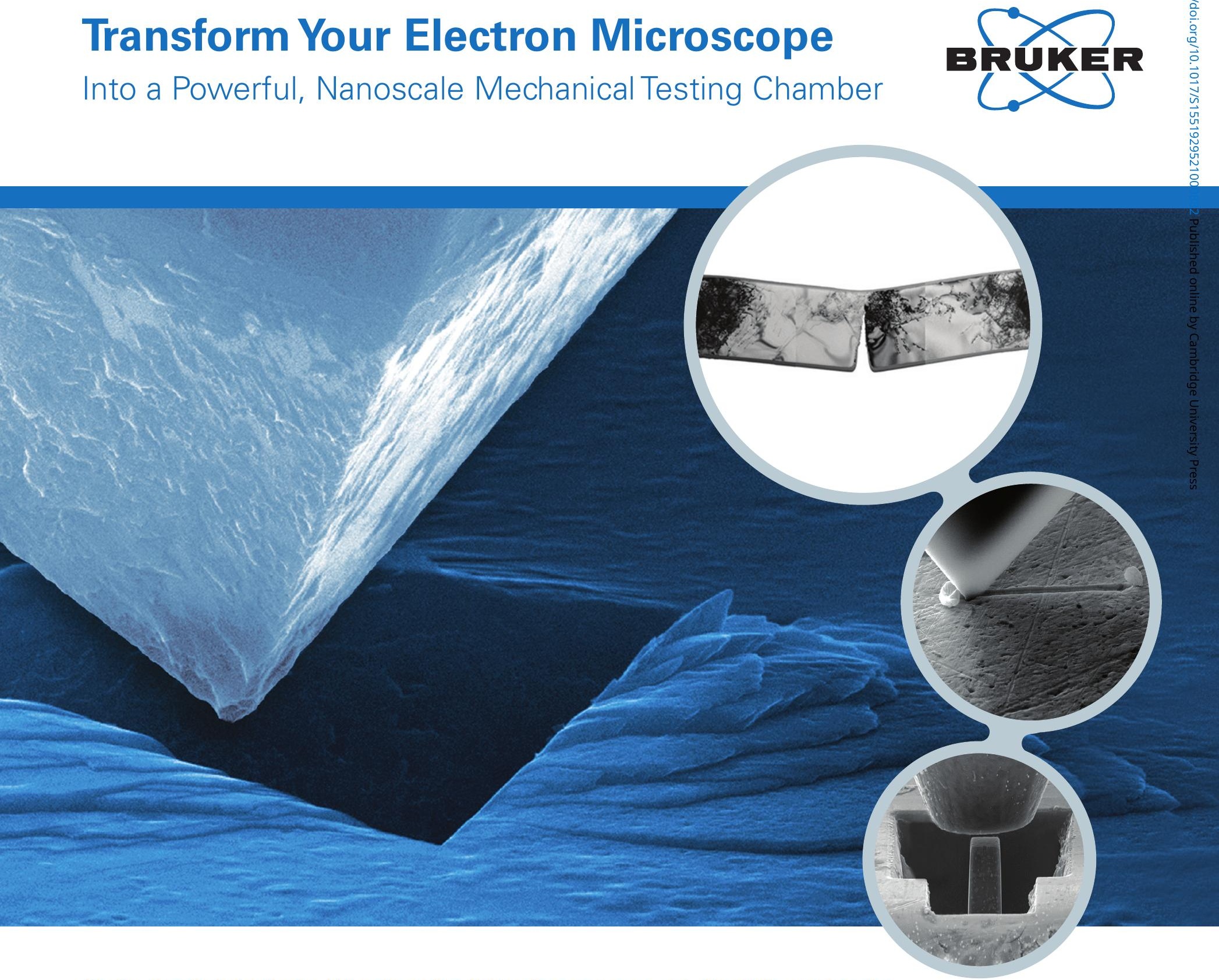

Gain Insights Into Mechanical Performance at the Nanoscale

Bruker's comprehensive suite of in-situ nanomechanical instruments combines powerful electron microscopy with small-scale mechanical testing. Each system is capable of a wide variety of testing modes, enabling unprecedented insights into nanoscale material behavior across the research spectrum.
- Nanoindentation
- NanoTribology
- Microbeam Bending
- High Temperature
- Pillar or Particle Compression
- Electrical
- Tensile Testing
- Dynamic and Fatigue

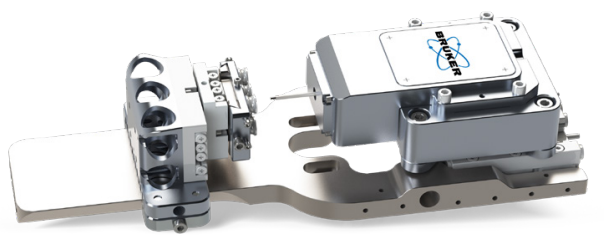

Hysitron PI 89 SEM

Picolndenter

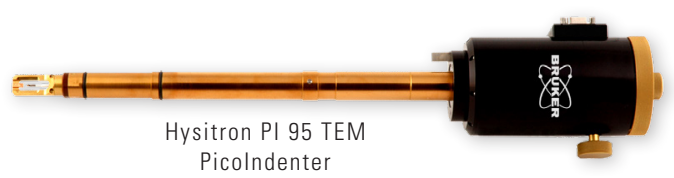

\section{口irita \\ www.bruker.com/picoindenters

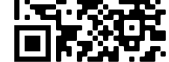

Innovation with Integrity 